\title{
A Critical Comparison of Approaches to Resource Name Management within the IEC Common Information Model
}

\author{
Nigel Hargreaves, Student Member, IEEE; Stefan Pantea; Gareth Taylor, Senior Member IEEE; \\ Malcolm Irving, Senior Member IEEE.
}

\begin{abstract}
Electricity network resources are frequently identified within different power systems by inhomogeneous names and identities due to the legacy of their administration by different utility business domains. The IEC 61970 Common Information Model (CIM) enables network modeling to reflect the reality of multiple names for unique network resources. However this issue presents a serious challenge to the integrity of a shared CIM repository that has the task of maintaining a resource manifest, linking network resources to master identities, when unique network resources may have multiple names and identities derived from different power system models and other power system applications. The current approach, using CIM 15, is to manage multiple resource names within a singular CIM namespace utilizing the CIM "IdentifiedObject" and "Name" classes. We compare this approach to one using additional namespaces relating to different power systems, similar to the practice used in CIM extensions, in order to more clearly identify the genealogy of a network resource, provide faster model import times and a simpler means of supporting the relationship between multiple resource names and identities and a master resource identity.
\end{abstract}

Index Terms-CIM, IEC61970, Interoperability, Model Repository, Resource Name Management, Smart Grid.

\section{INTRODUCTION}

$\mathrm{E}$ LECTRICITY networks have historically been controlled by power control systems connected through interfaces in a point-to-point arrangement. Due to the differences in the way application vendors have modeled the real network, these interfaces have often required custom development to suit individual power utility needs. With the emergence of smart grids the need for power system interoperability is seen as an essential component of the required flexibility to manage increasingly dynamic supply and demand scenarios [1]. Sharing of data between heterogeneous power systems and other applications forms the basis of control system

This work is funded and supported by National Grid Plc and the Engineering and Physical Sciences Research Council (EPSRC) for funding this research project.

N. Hargreaves is with Department of Engineering and Design, Brunel University, Uxbridge, UB8 3PH, UK (e-mail: nigel.hargreaves@brunel.ac.uk)

S. Pantea is with National Grid, Control Systems Development, Wokingham, RG415BN, UK (e-mail: stefan.pantea@nationalgrid.com)

G. Taylor is with Department of Engineering and Design, Brunel University, Uxbridge, UB8 3PH, UK (e-mail: gareth.taylor@brunel.ac.uk)

M. Irving is with Department of Engineering and Design, Brunel University, Uxbridge, UB8 3PH, UK (e-mail: gareth.taylor@brunel.ac.uk) interoperability [2]. This can be performed by abstracting data from within proprietary applications to a higher level supported by a common information model.

The power system data, now in the form of information models conforming to an international standard, promotes the exchange of information through vendor-agnostic interfaces to a wider number of systems and applications [3].

The opportunity to leverage the use of data previously siloed in databases, islanded in separate power systems, is a further attractive incentive for power utilities to adopt a common information model. However, the sharing and merging of information models across system interfaces and company boundaries presents the significant challenge of maintaining model integrity, through establishing model boundary authority, resource name and identity associations to a master identity [4][5]. This identity should be globally unique and persistent and relate to all instances of the same network resource, even though it may be referenced to a variety of names within different system models.

This paper critically compares approaches to maintaining resource identity and naming integrity, by referring to the use of IEC 61970 within the Common Information Model (CIM). In Section II we will describe the pertinent parts of the IEC CIM in more detail and in Sections III and IV present our critical comparison of the current IEC CIM approach to resource identity management by basing our argument upon experience of implementing a use case described in Section V, before drawing our conclusions in Section VI.

\section{THE IEC CIM}

The IEC Common Information Model (CIM), comprising IEC 61970, 61968 and 62325, [6] is regarded as central to a number of national and international smart grid standards frameworks [7] and forms the basis of one of five core smart grid interoperability standards identified by the National Institute of Standards and Technology (NIST) [8]. The CIM provides an object-oriented, scalable and extensible semantic reference model for modeling power system resources. IEC61970 was originally intended as a common information model to serve in mapping energy management system application programming interfaces (EMS-API) for transmission system operation but has grown to become a power utility domain ontology [9]. Successive releases of the CIM, driven by power utility information use cases, are 
continuously increasing its scope and resolution to support smart grid scenarios for power system interoperability and management.

To implement the CIM, the reality of the electrical grid is partitioned in resources that are modeled as instances of a particular Unified Modeling Language (UML) class. IEC 61970 CIM, as a standard ontology for power systems, offers multiple ways to model the real network. For example, network topology and connectivity models would use overlapping but different sets of UML classes in order to abstract the same reality for use in an operational context compared with a planning context. It is under such circumstances that the issue of resource naming and ID differences can arise, firstly at the power system model level and then at a higher level of abstraction within their corresponding common information models.

Network resource naming diversity and the possible need to manage multiple identities (IDs) are significant challenges to face when building a CIM model repository shared across an information infrastructure. Following a network resource's trajectory, starting from its UML inception and instantiations into particular CIM data models, presents a good opportunity to discover how the technology of the CIM model and its architecture are composed (Fig. 1).

The namespace is acknowledged as a fundamental part of a resource definition and trajectory [10]. It is used to uniquely identify instances of classes, types and their attributes inside a CIM model architecture. Objects serve two purposes: they promote understanding of the real world and provide a practical basis for computer implementation. In this context we are using the term object as a mechanism that reflects "the capabilities of the system to keep information about it (states), interact with it (interactions), or both" [11]. When new types of network resources, that are not included in a CIM standard model or profile are required to model a new information use case, the namespace concept is also used to identify and

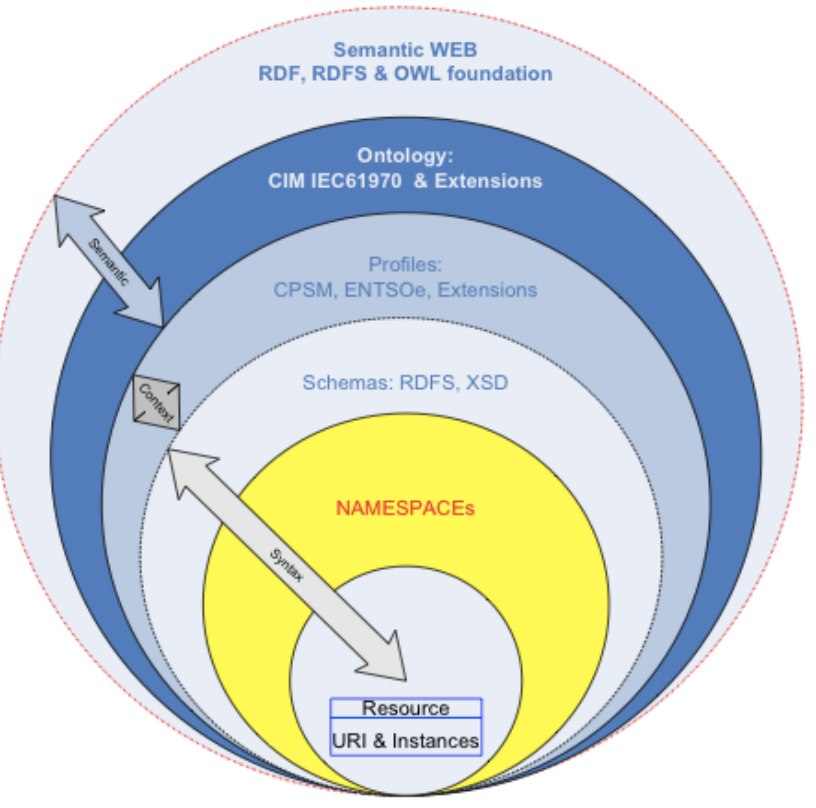

Fig. 1. Modeling layer reference framework, exemplifying the resource trajectory within a namespace. contain the CIM object extensions. We shall explain this further in the use case below. Namespaces are thus special objects as they contain sets of objects (states) and promote understanding of the real world by containing information about object interactions.

CIM model objects are reified at the instance level by "rdf:ID" statements. The namespace with the "rdf:ID" is the Uniform Resource Identifier (URI), the scope of which is usually provided by the parser within the system it is based. Thus the reference for the "rdf:ID" can be considered within the scope of a particular system and not normally globally unique, unless referenced as such. For this purpose the "rdf:ID” becomes the Master Resource ID ("mRID"), or part of it, for uniquely identifying objects when a model from one system is merged into another. Thus the power of resource identity stays in the "rdf:ID" and name, as a coupled concept. Multiple resource names can then be maintained even if identical as the "rdf:ID"s are unique. Identity management of resources comprises both "rdf:ID" and names, in a way that each one would not make sense without the other. The "rdf:ID" ensures uniqueness of the resource, and is intended to be used between non-human operators/agents, but names can take any value and are intended for human consumption.

Different names for the same resource can therefore coexist under the same "rdf:ID" statement either within different namespaces, or if all within the CIM namespace, through the use of additional UML classes. There are two options to update object names during a model merging process. For an existing resource, by the "rdf:about" statement, where the object is updated with more attributes or values, and for a new resource by using the "rdf:ID" statement. (Fig. 2)

A key aim of a shared model repository is to recreate a single, abstract reality from merged models in which use cases might require the containment of complex name structures and strategies. As the models composing this shared reality overlap and contribute their own view of object state and interaction the opportunities for confusion of object identityname coupling arise. For example, in instances when systems model identical network resources but their resource names and IDs are different.

In the next sections, we compare approaches to these challenges from the perspectives of current CIM methods and the proposed multiple namespace approach. We will discuss
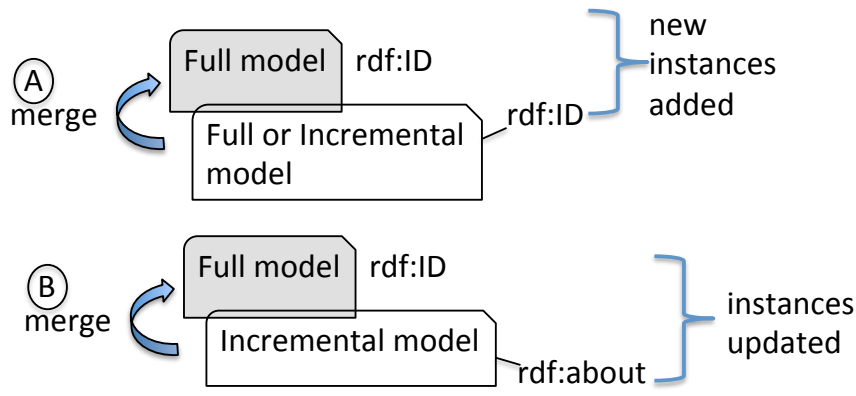

Fig. 2. Instance-level model merging. A: Two models composed of different objects add object IDs to the original model (in grey). B: Two models composed of the same objects. The incremental model updates the data and attributes within the first model but IDs remain unchanged. 
the advantages we see in the proposed approach compared to the present solutions provided within CIM 15.

\section{CURRENT CIM APPROACH TO RESOURCE IDENTITY MANAGEMENT (CIM 15)}

Resource identity management is a relatively simple task for a CIM data model exchanged between just two systems. However use of the CIM evolves rapidly from a point-to-point link, to a meshed multi-point data exchange in a smart grid power system applications context. Point-to-point data exchanges are transformed into a point-to-common data exchange type, as found in a shared model repository context. The shared repository model is composed from data models originating from different systems. In this context resource identity management becomes complex. The merged model increases in size, and in a short time, develops from a large to a very large model with millions of objects, amounting to a file size of multiple Gigabytes.

Network resources are often identified with heterogeneous names originating from within different power system models. For inclusion within an information model based on the IEC CIM they must be identified using the "IdentifiedObject" class. Attributes of this UML class are provided to develop the identity of a particular resource using human and machinereadable names. In early versions of CIM, up to the current standard, CIM 15, "IdentifiedObject" attributes, were provided as a solution to resource identity management. However this approach was seen to be limited in its effectiveness to handle multiple names for the same resource, originating within different power systems.

Due to these limitations, within release 15 of the IEC CIM, the "IdentifiedObject" class is revised to have less attributes (Table I) and additional classes, "Name", "NameType" and "NameTypeAuthority" were implemented for a better system of resource identity management. Each "IdentifiedObject" is now enabled to associate with 0 to $n$ "Name" classes. The "NameType" class is implemented to address instances where the same object may be given different names within the same or different power systems, similar to a 'local name' concept (Fig. 3). Thus, in order to capture the name-identity coupling in detailed naming strategies, CIM 15 decomposes its structure into several classes (at the UML conceptual level) which forces CIM adaptors to browse a more verbose instance model.
TABLE I

\begin{tabular}{|l|l|}
\hline \multicolumn{2}{|c|}{ Attributes of IdentifiedObject class with brief description - CIM 15 } \\
\hline aliasName & An alternative to the ".name" attribute \\
\hline mRID & A Master Resource ID issued by a Model Authority \\
\hline name & A name which may be non-unique \\
\hline
\end{tabular}

Table I. Attributes of "IdentifiedObject" class (CIM 15) with brief description of their function.

For large or very large CIM models this can imply lengthy parsing delays (in the order of several hours), even if only a relatively small section of the model's objects are required to be imported. If these objects were contained within more namespaces than the CIM master namespace however, it may be possible to selectively parse only the relevant sections of a model, corresponding to particular namespaces.

\section{NAMESPaCE APproach To Resource IDENTITY MANAGEMENT}

We propose a new solution to manage multiple resource names and identities using namespaces that would be of benefit within a merged-model repository context in the following ways:

A - Improved resource identity management by keeping power system genealogy of object name-identity coupling intact.

B - Improved model import and validation time when scaling from large to very large CIM models.

\section{A. Multiple resource identity management}

We assume that each power system would require only one name per resource. We view namespaces as a container for modeled resource objects within their respective power system. The solution then uses one additional namespace for every power system that connects to the shared repository model, similar to the way namespaces are used to define CIM extensions. An example of CIM XML is presented in Fig. 4 to illustrate the containment of the name-identity coupling within separate namespaces as two models are merged. Due to space limitations only small excerpts of code are given to illustrate the principle. The native CIM class, "IdentifiedObject", will be qualified in namespaces defined by a system's CIM adapters. In this way, resources names will have their system genealogy explained, which is a significant benefit in a shared

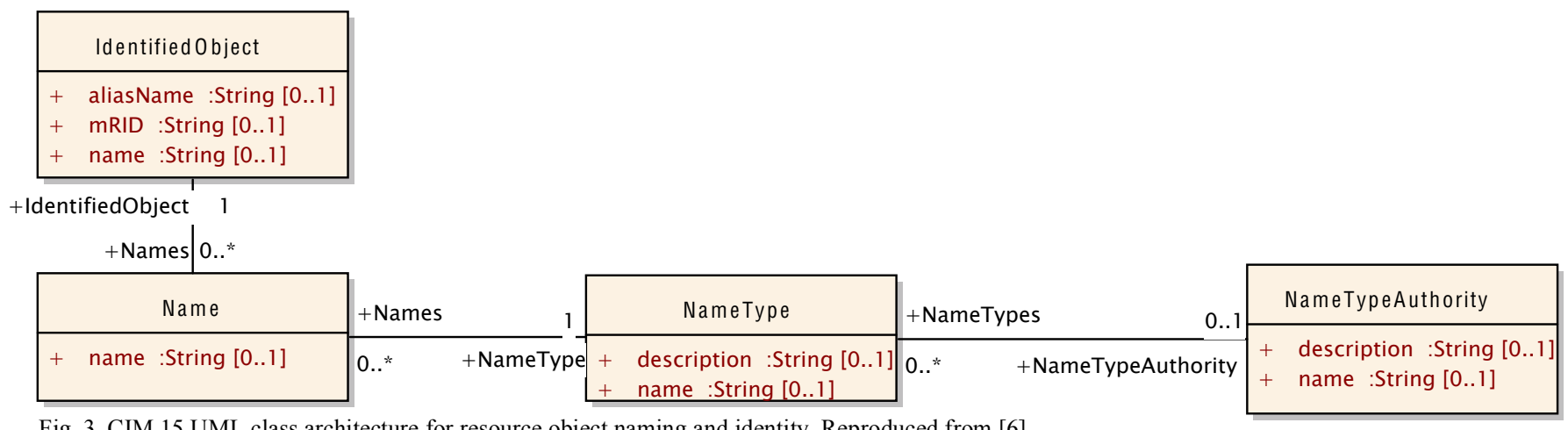

Fig. 3. CIM 15 UML class architecture for resource object naming and identity. Reproduced from [6] 
By using namespace filters however, the opportunity for import time performances to be optimized over current practices may be possible. Objects qualified in namespaces relevant for that particular CIM adapter would be imported and the rest ignored by the filter. For a small CIM data model import speed improvements are not significant but for large and very large models this would offer greater time savings.

\section{USE CASE}

The authors base the use case on a practical application of the CIM within National Grid (NG), the GB Transmission System Operator. The NG EMS system has telemetry points for the majority of the synchronous machines connected into the high voltage network. GB market requirements package generation capacity as balancing mechanism units (BMUs), whose identity is stored within the NG Registration Database (RDB). The following use case concerns merging the data about the synchronous machines from the EMS with their BMU definitions held in the RDB in order to inform a third system, the Data Historian, which records operational records for sharing with other NG systems (Fig. 6). To model the BMUs, it was also necessary to extend the CIM so that the NG-specific RDB data could be merged into the larger EMS model. The relationship between the BMU and telemetry measurements, analog values, is generally of an "n to n" type. For each BMU we can have none or many analog points.

With a CIM repository architecture, implementing the BMU concept requires merging an incremental BMU CIM model with the EMS model, to achieve the necessary BMUsynchronous machine and measurement associations. To avoid potential collisions with existing "rdf:IDs" of the telemetry points the BMUs telemetry points have slightly different "rdf:IDs" but the same name. Consistency of names is later used by the Data Historian system, into which the merged BMU-EMS model is imported, for measurements to be matched with real time data updated via Inter-Control Center Communications Protocol (ICCP) every 6 seconds. By

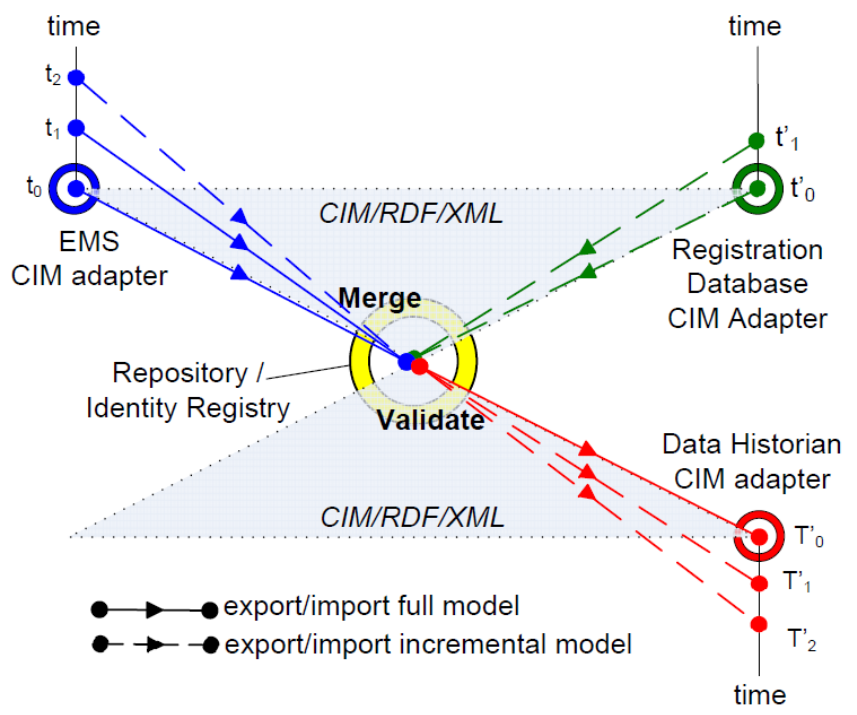

maintaining separate namespaces to contain both BMU and EMS model objects the identity of resources, coupled in the "rdf:ID" and name, stays intact. CIM 15 breaks down this synergy however, as explained above, as well as adding new classes that are supposed to facilitate naming management.

The proposed approach of using different namespaces contrasts favourably with the approach supported by the IEC CIM, in the way it keeps the name-ID genealogy intact within separate namespaces, as well as introducing less additional code to describe the naming classes within the merged model, which incorporates a set of new classes, requiring complex mapping to resource names.

\section{CONCLUSIONS AND FURTHER WORK}

This paper addresses efficient power network resource identity management to minimize costs when scaling from large to very large CIM data model merging contexts. It suggests advances that would benefit users of different releases of the IEC Common Information Model without costly modification to power system CIM adaptors. It furthers the objective of multiple merged-model integrity as would be required within utility and regional shared model repository scenarios by making the following contributions:

- Innovative resource identity management using namespaces, preserving name-ID genealogy.

- Improved CIM adaptor time performance for CIM model imports through namespace filters, by ignoring unqualified namespaces.

- Potentially reduces costs of building a shared common model repository, as with the proposed modeling approach, resources name authorities within CIM15 may not be required.

- In the context of a shared model repository a third party identity management system may not be required since this intelligence would be included within the namespace;

Further work will include the demonstration of namespace filters at power system CIM interfaces in order to facilitate the separation of available data within a shared, merged CIM model. Such an approach will then enable only the data that is required by that system to be imported and the rest ignored.

\section{ACKNOWLEDGEMENTS}

National Grid Electricity Transmission (NGET) working in collaboration with Brunel University commissioned this research as part of the Brunel Industrial Doctorate Scheme (BIPS). The authors would like to acknowledge the valuable insights for this paper offered by Mr. Ian Hornby of the National Grid Operational Strategy Development team. This paper was published with permission from National Grid. The opinions expressed in this paper are that of the authors and not that of National Grid.

Fig. 6. Schematic representation of NG multiple system modelmerging use case. 


\section{REFERENCES}

[1] N. Hargreaves, G. Taylor, A. Carter, "Information Standards to support Application and Enterprise Interoperability for the Smart Grid," in Power \& Energy Society General Meeting, 2012. PES '12. IEEE, 2012, [accepted].

[2] R. Ambrosio and S. Widergren, "A framework for addressing interoperability issues," in Power Engineering Society General Meeting, 2007. IEEE, 2007, pp. $1-5$.

[3] D. Becker and T. L. Saxton, "CIM standard for model exchange between planning and operations," in Power and Energy Society General Meeting - Conversion and Delivery of Electrical Energy in the 21st Century, 2008 IEEE, 2008, pp. 1-5.

[4] J. P. Britton, "Designing model exchange processes with CIM and 'RMA sets'," in Power Systems Conference and Exposition, 2006. PSCE '06. 2006 IEEE PES, 2006, pp. 487-489.

[5] N. Hargreaves, G. Taylor, A. Carter and A. McMorran, "Developing emerging standards for power system data exchange to enable interoperable and scalable operational modelling and analysis," Universities' Power Engineering Conference (UPEC), Proceedings of 2011 46th International, pp. 1-5, 2011.

[6] CIM Users Group,

"iec61970cim15v33 iec61968cim11v13 iec6232501v07" [Online]. Available: http://cimug.ucaiug.org/CIM\%20Model\%20Releases/Foms/AllItems.asp $\mathrm{x}$

[7] M. Uslar, M. Specht, S. Rohjans, J. Trefke, J. M. Vasquez Gonzalez, "Examples of Using the CIM," Springer-Verlag, Berlin Heidelberg, 2012, p31.

[8] National Institute of Standards and Technology (NIST), "NIST Identifies Five 'Foundational' Smart Grid Standards". [Online]. Available: http://www.nist.gov/public affairs/releases/smartgrid 100710.cfm

[9] S. A. Neumann and T. D. Nielsen, "CIM interoperability challenges," in Power and Energy Society General Meeting, 2010 IEEE, 2010, pp. 1-5.

[10] Xiaofeng Wang and S. Van Ausdall, "Representing business data semantics in CIM using UML," in Power Systems Conference and Exposition, 2006. PSCE' '06. 2006 IEEE PES, 2006, pp. 480-486.

[11] S. Bennett, S. McRobb, R. Farmer, "Object Oriented System Analysis and Design using UML", 1999, pp62-63.

[12] CIMdesk is a product of Power Info LLC, USA. [Online]. Available: http://www.powerinfo.us/opensource/cimspy.html

\section{BIOGRAPHIES}

Nigel Hargreaves (Student M' 10) Obtained his MSc. In Climate Change Impacts and Sustainability from Brunel University, London in 2008 and his BSc. in Air Transport Engineering from the City University, London in 1981. $\mathrm{He}$ is currently studying for his $\mathrm{PhD}$. in Electrical Engineering while working within the Control Strategy Development team at National Grid. His area of interest is Enterprise Information Modeling and Management.

Stefan Pantea (IET'12) is an Advanced Application Engineer within the Future Control Systems in the National Grid, UK. He is assigned to working on the Common Model Information for network operational systems. He has 10 years international SCADA experience in Substation Supervisory Control Systems (SCS), Distribution Management (DMS) and Energy Management Systems (EMS). His area of interest is Information Models Management.

Gareth A. Taylor (SM' 06) received his BSc degree from the University of London, UK in 1987 and MSc and PhD from the University of Greenwich, UK in 1992 and 1997, respectively. He was the National Grid UK postdoctoral scholar at Brunel University, UK from 2000-2003. He is currently a reader and course director within the Brunel Institute of Power Systems, Brunel University, UK. His research interests include power system and network optimization.

Malcolm Irving (SM' 93) is Professor of Power Systems in the School of Engineering and Design at Brunel University, Uxbridge, UK. He is also Codirector of the Brunel Institute of Power Systems, a major research group in the University specialising in algorithms and software for generation, transmission and distribution systems. He is the author or co-author of more than 150 research publications in the fields of power network analysis and optimal power system operation. 